\title{
Characteristic features of water dynamics in restricted geometries investigated with quasi-elastic neutron scattering
}

\author{
N. C. Ostia ${ }^{\mathrm{a}, 1}$, A. Cotéa,1,2, E. Mamontova ${ }^{\mathrm{a}}$, A. Ramirez-Cuesta ${ }^{\mathrm{a}}$, D.J. \\ Wesolowski ${ }^{b}$, S.O. Diallo ${ }^{\mathrm{a}, *}$ \\ ${ }^{a}$ Chemical and Engineering Materials Division, Oak Ridge National Laboratory, Oak \\ Ridge, TN 37831 \\ ${ }^{b}$ Chemical Sciences Division, Oak Ridge National Laboratory, Oak Ridge, TN 37831Oak \\ Ridge, TN 37831
}

\begin{abstract}
Understanding the molecular behavior of water in spatially restricted environments is key to better understanding its role in many biological, chemical and geological processes. Here we examine the translational diffusion of water confined to a variety of substrates, from flat surfaces to nanoporous media, in the context of a recently proposed universal scaling law [Chiavazzo et al., Nat. Comm. 5, 1 (2014)] [1]. Using over a dozen previous neutron scattering results, we test the validity of this law, evaluating separately the influence of the hydration amount, and the effects of the size and morphology of the confining medium. Additionally, we investigate the effects of changing instrument resolutions and fitting models on the applicability of this law. Finally, we perform quasi-elastic neutron scattering measurements on water confined inside nanoporous silica to further evaluate this predictive law, in the temperature range $250 \leq T \leq 290 \mathrm{~K}$.
\end{abstract}

Keywords: water diffusion, porous media, neutron scattering

\footnotetext{
*To whom correspondence should be addressed Email address: omardiallos@ornl.gov (S.O. Diallo)

${ }^{1}$ Contributed equally to this work

${ }^{2}$ Permanent address: Department of Physics, University of Miami, Coral Gables, FL 33124
} 


\section{Introduction}

Although water has been at the center of considerable research efforts over the years [2-5], its peculiar but important thermo-physical properties are far from being fully understood. Compared to non-hydrogen bonded liquids, water behaves in some respect more like a solid due to its strong hydrogen bond network and exhibits key properties relevant to many biological, chemical and technological processes like unusually large specific heat, high melting point, and large surface tension. Understanding how water molecules behave in various spatially restricted environments is of key scientific importance in geophysics, biological physics and many other research areas. For this reason, the diffusive motions of water molecules nanoscopically confined in materials like porous silica have been widely investigated using a variety of probes including neutron scattering [6-9, 9-12], dielectric spectroscopy [2] and NMR [13-15]. These studies have revealed a clear retardation of the water diffusion compared to the bulk phase, which becomes increasingly pronounced as the restrictive dimension or the temperature decreases. However, the difference in size, geometry and morphology of the pores, the instrumentation used, and the various analysis methods have often introduced ambiguity in the interpretation or reconciliation of the results. These issues can be overcome by conducting experiments in which only one experimental parameter (temperature, pore size, morphology, etc.) is changed at a time. This ideal approach would require years of planning and measurements to complete with neutron scattering, because of the limited availability of the neutron sources. Alternatively, it may be convenient to examine the influence of each variable separately by using existing measurements performed over many decades by different research groups.

In this article, we thus present a survey of previous quasi-elastic neutron scattering (QENS) measurements of water diffusion, examined in the context of a predictive scaling law for water transport, recently proposed by Chiavazzo et al. [1]. Our aim is to look for identifiable trends or features in the water dynamics under given confinement conditions. Using over a dozen QENS results, we test the validity of this law, evaluating separately the effects of variable hydration, changing confinement size and dimensionality. This scaling depends primarily on a single parameter $\theta$, the ratio between the average number of water molecules that are most influenced by the surface or pore walls and the total number of water molecules under confinement. This parameter can also be related to the fractional volume occupied by these 
molecules compared to the total volume available. This geometry-dependent parameter tends to be smaller for systems with large confining volume or small curvature at higher coverages. Based on the aforementioned model, the translational diffusion coefficient $D_{r}$ of confined water, which is typically measured with neutrons, can be conveniently expressed at any temperature $T$ above the bulk water nucleation temperature as,

$$
D_{r}(T)=\theta D_{c}+(1-\theta) D_{b u l k}(T) .
$$

Here $D_{b u l k}(T)$ represents the bulk water translational diffusion at temperature $T$ and $D_{c}$ the temperature independent translational diffusion coefficient of the water molecules that are solely affected by the walls. In practice, $D_{\text {bulk }}(T)$ can be obtained from the literature [16], and $D_{c}$ and $\theta$ are specific to the confining sample and its geometrical characteristics. Because Eq. 1 assumes that $\theta$ and $D_{c}$ do not change significantly with temperature, its validity can only be verified over temperature ranges where water does not experience phase changes, and for which $D_{c}$ and $\theta$ indeed remain constant on average. Our empirical observation is that Eq. 1 could be reliably verified for water confined in a number of confining media at temperatures between $\sim 250 \mathrm{~K}$ and room temperature. The low limit is set by the homogeneous nucleation temperature of bulk water $\left(T_{H} \simeq 230-235 \mathrm{~K}\right)$ and the high limit by the accessible energy window of the neutron instrument (approximately around $300 \mathrm{~K}$ for most existing high energy backscattering spectrometers).

This article is organized as follows. Sections 2, 3 and 4 evaluate respectively the influence of hydration, confinement size, and morphology on the scaling transport law described above. We then discuss the importance of hydrophilicity/hydrophobicity as well as the impact of varying instrument resolution and data modeling on this law in sections 5 and 6 . In section 7 , we use exact geometrical models to better quantify water adsorption based on surface morphology and hydration amount. Section 8 examines in details the pore size dependence of water dynamics in folded mesoporous silica (FSM) materials, with cylindrical pores. Our final remarks are presented in section 9 .

\section{Hydration dependence}

Here we investigate the applicability of Eq. 1 under varying hydration conditions. The goal is to look for systematic evolution or patterns in the 
characteristic variables $\theta$ and $D_{c}$ as the hydration level, defined here as the fractional mass of water uptake per mass of sample, $h=m_{\text {water }} / m_{\text {sample }}$, is increased for a given substrate. Although this so-defined hydration parameter $h$ does not necessarily correspond to the filling ratio $V_{\text {fill }} / V$, where $V_{\text {fill }}$ and $V$ are respectively the filled volume and the total confining volume, it can be used to estimate it, provided the sample characteristics (such as porosity, geometry, density) and water layer densities are known. For example, using the dry density of Vycor glass of $1.45 \mathrm{~g} / \mathrm{cc}$ and the porosity of $28 \%$ and a layer-by-layer filling of the $50 \AA$ cylindrical pore of Vycor at constant density, Bellissent-Funel et al. [17] estimated full pore filling to correspond to $100 \%$ water uptake, $\sim 3-4$ layers of water on the internal surface to $52 \%$ hydration, and a monolayer to $25 \%$ hydration. Using the $D_{r}$ reported by BellissentFunel and co-workers as a starting example, we plot them in Fig. 1 as a function of $D_{b u l k}(T)$ reported by Teixeira et al. [16]. The $D_{r}(T)$ values are for water in porous $50 \AA$ Vycor at respective hydration levels of (weight \%) $25 \%, 52 \%$ and $100 \%$. These plots allow us to extract the corresponding $\theta$ and $D_{c}$, based on the intercept and slope of the fits (solid lines). Using imposed constraints $0 \leq \theta \leq 100 \%$ and $0 \leq D_{c} \leq D_{b u l k}$, the validity of the scaling expression above can be tested. We find in the case of Vycor that $D_{c}$ increases with increasing hydration level $h$ while $\theta$ follows the opposite trend. This observation is also true for water in a number of other systems such as protein, zeolite and hydrotalcite clay, as indicated in Table 1 and can be generalized. We will return to this point in section 4 .

\section{Confinement size}

While it is generally well established that tighter confinement leads to slower dynamics, it is is not clear how variable confinement dimension would affect the parameters in Eq. 1. We thus examine the validity of the scaling relation using data from the well-characterized silica system MCM-41 with variable pore sizes, as previously investigated by Takahara et al. [21] on the MIBEMOL spectrometer at the Laboratoire Léon Brillouin (France). We verified that the law can indeed be applied, yielding in general a $\theta$ that decreases with increasing pore size (or looser confinement), and a $D_{c}$ that increases, as exemplified by Fig. 2. For a given system, the lower limit of this range appears to be set by the neutron instrument used, yielding much lower values with the higher energy resolution spectrometers. The dependency of $\theta$ and $D_{c}$ on pore size found in MCM-41 is consistently similar across all the 


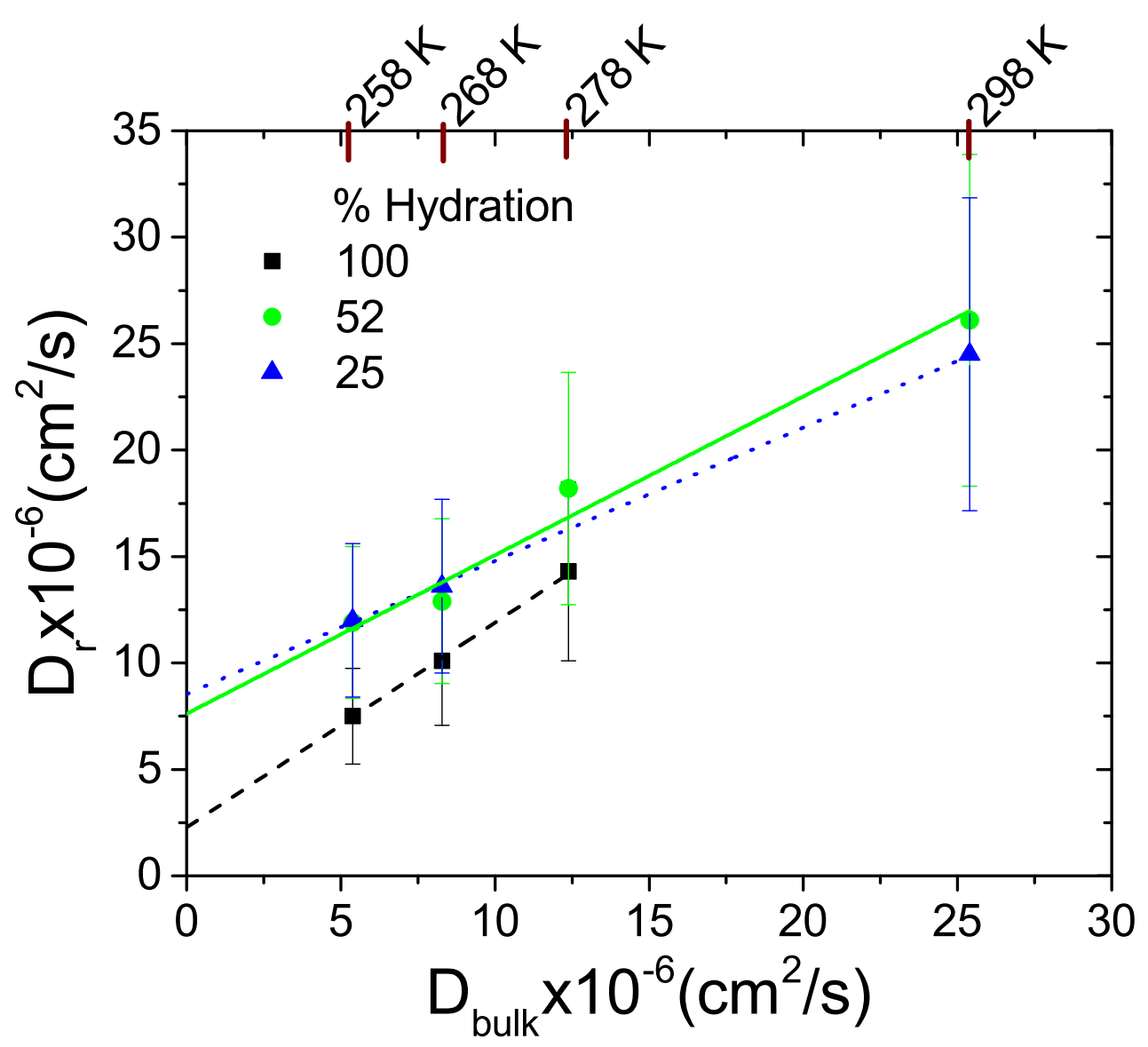

Figure 1: Hydration dependence (measured as weight \%) of water diffusion $D_{r}$ vs $D_{b u l k}$ in porous Vycor glass (50 A average pore dimension). Data points correspond to hydration level of $25 \%$ (blue triangle), $52 \%$ (green circles) and $100 \%$ (black squares). The lines are fits of Eq. 1 to the data Refs. [16, 17]. 
Table 1: Best fit parameters of Eq. 1 using reported $D_{r}$ values from selected materials ([17-20] as a function of increasing water uptake $h$ (weight \%). $h$ can be used to estimate the filling fraction, given the sample density, porosity, the confinement geometry and dimension.

\begin{tabular}{|c|c|c|c|}
\hline \hline Sample & $h=m_{\text {water }} / m_{\text {sample }}$ & $\theta$ & $D_{c}\left(10^{-6} \mathrm{~cm}^{2} / \mathrm{s}\right)$ \\
\hline Vycor [17] & $25 \%$ & $37 \%$ & 22.7 \\
& $52 \%$ & $25 \%$ & 29.8 \\
& $100 \%$ & $4 \%$ & 59.4 \\
\hline Zeolite [18] & $22 \%$ & $86 \%$ & 0.09 \\
& $28 \%$ & $85 \%$ & 0.95 \\
\hline Hydrotalcite clay [19] & $10 \%$ & $78 \%$ & 2.36 \\
& $30 \%$ & $4 \%$ & 75.3 \\
\hline Lysozyme [20] & $35 \%$ & $77 \%$ & - \\
& $45 \%$ & $54 \%$ & - \\
\hline \hline
\end{tabular}

systems we examined. It is the case for the activated carbon fibers [22] for example and for many other porous media, as listed in Table 2. We conclude that this trend with size is thus a generic feature of confined water.

In one of particular cases examined, that of the fully hydrated MCM-41 [23] measured on the HFBS instrument, we have not been able to extract meaningful $D_{c}$ and $\theta$ values. This exception highlights the difficulty associated with comparing data collected from various instruments or analyzed using different models. We will return to this particular point in section 8.2.

\section{Confinement morphology}

In this section, our aim is to investigate how the parameters in Eq. 1 evolve with changing confining morphology or geometry. Since the dimensional flow of water $(1 \mathrm{D}, 2 \mathrm{D}$ or $3 \mathrm{D}$ flow) in tight confinement depends primarily on the confining morphology, we found it convenient to categorize the various host materials using those criteria. With this simple classification, we have surveyed water diffusing in $2 \mathrm{D}$ or two-dimension primarily on planar or between layered surfaces, in 1D using observations based on narrow unconnected pores and finally in 3D using reports from materials with interconnected pore network. The findings for each category are summarized below. 


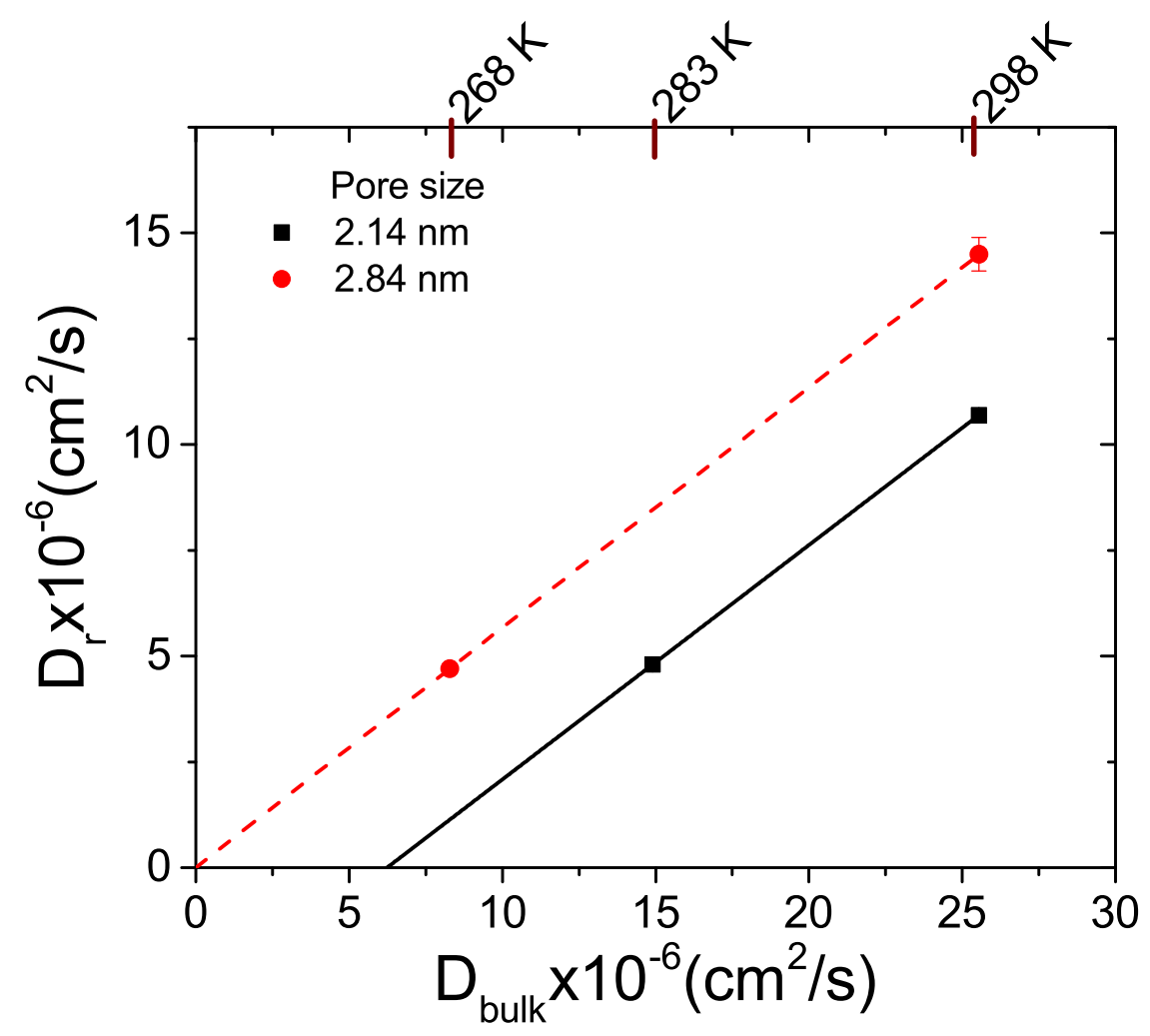

Figure 2: Water diffusion coefficient $D_{r}$ vs $D_{\text {bulk }}$ in nanoporous silica MCM-41 for two different pore sizes, respectively 21.4 and $28.4 \AA$ diameter. The lines are the best fits of Eq. 1. Data from Refs. [21] and [16]. 


\subsection{Planar or intercalated surfaces - 2D diffusion}

We begin by investigating the characteristic features for the simple case of water adsorbed on a surface. As a representative example for this, we chose the commonly studied hydration layer on proteins. Water around proteins has been known to exhibit much slower dynamics compared to the bulk phase, with some ten-to-fifty fold decrease in diffusivity [24]. Using data taken on MIBEMOL with C-phycocyanin protein [25], we verified the scaling law, obtaining $\theta=64 \%$ and $D_{c} \simeq 5.6 \times 10^{-6} \mathrm{~cm}^{2} / \mathrm{s}$. Using a more recent QENS report investigating the connection between lysozyme dynamical transition and hydration water by Wang et al. [20], we found $\theta$ to decrease from a value of $74 \%$ down to $54 \%$ as the hydration level is increased from 20 weight $\%$ up to about $45 \%$, but could not extract meaningful values for $D_{c}$. To get these results, it was necessary for us to first estimate $D_{r}$ from [20] using $D_{r}=\frac{l^{2}}{6 \tau}$, where $l=2 \pi / Q$ at $Q=0.5 \AA^{-1}$ since $D_{r}$ were not explicitly provided. We thus anticipate the $\theta$ and $D_{c}$ values to change with the method used to estimate $D_{r}$ but the overall trend of the characteristic parameters $\theta$ with increasing hydration to remain the same. While there are many other excellent QENS studies of protein hydration water, a good number of them $[26,27]$ are focused on clarifying the role water dynamics plays on the dynamical transition, and not so much on the diffusion of water itself. Therefore, the few examples provided above are simply a representative snapshot of cases where we could directly extract $D_{r}$ without much further data processing.

We also investigated the case of layered structured host minerals, such as hydrotalcite [19] as indicated in Table 1, and Na- and Ca-montmorillonite $[19,28]$. In the montmorillonite case, we get $\theta$ in the range $45-50 \%$ and $D_{c}$ between $3-4 \times 10^{-6} \mathrm{~cm}^{2} / \mathrm{s}$. Including other examples such as Na-vermiculite clay [29] for which $\theta=84 \%$ and $D_{c}=5.1 \times 10^{-6} \mathrm{~cm}^{2} / \mathrm{s}$, we conclude that the law can be applied for most $2 \mathrm{D}$ environments.

\subsection{Nanoporous materials - case of $1 D$ and $3 D$ diffusion}

The well-ordered porous structure is by far the most widely investigated pore structure with neutrons, in part because it is readily available, enables systematic size dependence studies, and is easy to model in molecular dynamics simulations. The most notable example of ordered pore network is that having a 2D hexagonal arrangement of cylindrical pores[21, 30, 31], such as MCM-41, FSM or SWNT. The nanometer size pores enables studies of water diffusion along the 1D-like channels. In all of these nano-channels, we found 
that on average approximately $50-80 \%$ of the adsorbed water molecules are directly influenced by the walls with a retarded mobility compared to the rest of the molecules that act like bulk water. For example, we found $\theta=63 \%$ and $D_{c}=5 \times 10^{-6} \mathrm{~cm}^{2} / \mathrm{s}$ in untreated $14 \AA \mathrm{SWNT}$ pores, compared to $\theta=45 \%$ and $D_{c} \sim 0$ in $21 \AA$ pore MCM-41 pores. Comparable results were obtained for water in the micropores of FSM materials, as detailed below in Section 8. Similarly, we find $\theta=94 \%$ and $D_{c}=0.8 \times 10^{-6} \mathrm{~cm}^{2} / \mathrm{s}$. Comparing with the cylindrical SWNT or MCM-41, the amount of surface water is definitely greater in the rectangular channels. This can be attributed primarily to the tighter $7 \AA$ confinement in the rectangular channel rather than to the difference in geometry.

Excluding a few exceptions, we have verified the scaling law for a number of well-ordered pore materials, confirming its applicability on these systems, concluding again that $\theta$ is reduced and $D_{c}$ is increased with larger water content.

In natural confinement situations like in rocks or bones, water is constrained to flow within randomly arranged cavities of random connectivity and heterogenous size distribution. These situations can be experimentally simulated using well known porous silica such as vitreous Vycor, an interconnected pore network with a relatively broad size distribution. As stated in section 2, we found the scaling law to hold in Vycor as well as in other randomly arranged pore structures such as the Zeolite [18], and the Nafion membrane $[11,32]$, leading respectively to $\theta=85 \%$ and $83 \%$ and to $D_{c} \simeq 10^{-6}-10^{-7}$ (depending on the hydration level) and $12.5 \times 10^{-6} \mathrm{~cm}^{2} / \mathrm{s}$ in each case. Similarly, we find that for water confined within the interior volume of unconnected nanometer size spherical shells such as in the reverse Aerosol OT micelles or AOT [33] measured on the IN5 instrument, the majority of the confined water is surfactant bound, with $\theta=96 \%$ and $D_{c}=1.1 \mathrm{x}$ $10^{-6} \mathrm{~cm}^{2} / \mathrm{s}$. With increasing core radius, the water in the micelles eventually behaves much like bulk water.

For calcium-silicate hydrate (C-S-H, the main product of the hydration of Portland cement), however, we found the scaling to work only above a certain hydration threshold of approximately 30\%. Below this level, at for example 10 or $17 \%$ as investigated on the BASIS spectrometer [34], the diffusion coefficients show no clear dependence on the bulk water data. The $30 \%$ hydration data yields a large fraction of water molecules influenced by the confining surfaces $(\theta=95 \%)$, with a corresponding retarded diffusivity with $D_{c}=0.35 \times 10^{-6} \mathrm{~cm}^{2} / \mathrm{s}$. Just like in the case of Aerosol OT reverse 
micelles, the observed values in C-S-H suggest that only a small fraction of the molecules behave macroscopically like bulk water (since $\theta \neq 100 \%$ ). This however does not happen before all chemically active sites necessary for water bindings in the cement paste become saturated with water. Once this is fulfilled, the starting large value of $\theta$ (95\%) observed at $30 \%$ hydration will then start to decrease as the hydration is increased towards the bulk amount, consequently increasing $D_{c}$ gradually until it is no longer defined in the bulk phase where $\theta=0$.

The key observation regarding morphology as per the classification above is that at full water fillings of either the surface or the pore, the geometrical parameter $\theta$ experiences a decrease with reduced dimensionality, certainly going from $3 \mathrm{D}$ to any lower dimension. The $2 \mathrm{D}$ to $1 \mathrm{D}$ crossover is less conclusive based on the data investigated but we believe that the variation of $\theta$ with geometry is largely set by the surface area offered by that geometry. In this event, we expect the same confinement size to lead to a larger $\theta$ value for spherical geometry (3D) than for intercalated gap (2D). The $1 \mathrm{D}$ case (represented by the tight cylindrical pores) would yield $\theta$ values that are in between the 3D and 2D case.

The diffusion parameter $D_{c}$ on the other hand does not exhibit any particular trend with different morphology. It seems to ultimately converge towards $D_{\text {bulk }}$ as the $3 \mathrm{D}$ case is approached (as the confinement dimension increases to macroscopic length scale). Unlike $\theta$, the magnitude of $D_{c}$ seems to correlate with the instrument resolution, yielding smaller values for the higher resolution machines.

The parameter $d=2 R$ in Table 2 corresponds to the characteristic confinement dimensions depicted in Fig. 3 for each of the three main geometries. It refers to diameter in the case of cylindrical (1D) or spherical confinement (3D), and to the slit dimension in the case of slit-geometry. For a single planar surface, a confining gap cant be defined, as in the above three cases. In this case, it is reasonable to use the confining radius $a$ derived from fits to EISF (elastic incoherent structure factor to which we return below), as the nominal confining dimension. This measured parameter gives a good estimate of the distance over which the surface does appreciably influence the adsorbed water. 
Table 2: Overview of various confining media (shell: spherical shell, rand. arr. : randomly arranged cavities, ord. cyl. (or rect.) : ordered cylindrical (or rectangular) tubes, rand. slit. : randomly arranged slits) as obtained from the literature for which the scaling law was tested against for $245 \leq T \leq 300 \mathrm{~K}$. The data shown is by no means exhaustive, but a representative snapshot of existing studies where temperature dependent translational diffusion coefficient is either explicitly reported or tabulated. The confining size or characteristic dimension $d$ and instrument resolution resolution (Res.) are respectively in units of $\AA$ and $\mu \mathrm{eV}$.

\begin{tabular}{|c|c|c|c|c|c|}
\hline \hline Media & $\mathrm{d}(\AA)$ & Morph. & Instrument & Res. $(\mu \mathrm{eV})$ & Model \\
\hline Protein [25] & $4-5$ & planar & MIBEMOL & 28 & LOR. \\
Protein [20] & $4-5$ & planar & BASIS & 3.5 & KWW \\
\hline Clay [19] & $14-18$ & layered & FOCUS & $45-145$ & LOR. \\
Clay [28] & $10-16$ & layered & FOCUS & $45-145$ & LOR. \\
\hline AOT [33] & $7-12$ & shell & IN5,BASIS & $3.5-25$ & LOR. \\
& & & IN16 & & \\
\hline Vycor [17] & 50 & rand. arr. & MIBEMOL & 28 & LOR. \\
C-S-H [34] & $10-30$ & rand. arr. & BASIS & 3.5 & KWW \\
Nafion [32] & 14 & rand. arr. & HFBS & 0.8 & LOR. \\
Zeolite [18] & $11-14$ & rand. arr. & IN5 & 11 & KWW \\
\hline MCM-41 [21] & 28 & ord. cyl. & MIBEMOL & 28 & KWW \\
MCM-41 [21] & 21 & ord. cyl. & MIBEMOL & 28 & KWW \\
SWNT [30] & 14 & ord. cyl. & HFBS & 0.8 & LOR. \\
FSM-12 & 16 & ord. cyl. & BASIS & 3.5 & KWW \\
FSM-22 & 39 & ord. cyl. & BASIS & 3.5 & KWW \\
\hline Cu Rub. [35] & 7 & ord. rect. & HFBS & $1-120$ & KWW \\
& & & AGNES & & \\
\hline ACF-10 [22] & 12 & rand. slit & BASIS & 3.5 & KWW \\
ACF-20 [22] & 18 & rand. slit & BASIS & 3.5 & KWW \\
\hline \hline
\end{tabular}




\section{Hydrophilicity}

The scaling law is ideally suited to describe water diffusion in totally hydrophilic systems where the interaction between the water and the confining surfaces is known to be significant, and for which $\theta$ is non-zero. In these cases, $D_{c}$ tends to be significantly smaller compared to bulk and in some cases zero. We also found this scaling law capable of describing water in other confining media that would otherwise be totally hydrophobic if not for their hard-to-remove surface functional groups such as in untreated carbon materials (e.g. ACF, SWNT). These incidental situations are particularly interesting, as they can represent relevant situations in biological macromolecules where both hydrophilic and hydrophobic groups tend to coexist. We have satisfactorily applied the scaling law in mixed hydrophilic and hydrophobic ACF samples [22] with varying slit-shaped pores. We have followed the same analysis procedure to evaluate the water dynamics in totally hydrophilic silica FSM samples, which we describe in detail below. Our conclusion is that the scaling law can be applied to confined water almost universally, so as long as the confining medium is not totally hydrophobic.

While in most cases, we found the scaling relation to hold, we observed some limited or non-applicability in a few cases. As already mentioned earlier, this is the case for example for the surface modified MCM-41 system [36], where the best fit to the data lead to a reasonable $\theta=12 \%$, but not to any physically meaningful value for $D_{c}$, giving $D_{c} \leq 0$ within the error. Constraining $D_{c}$ to zero or positive in the fits only made them worse, leading to a somewhat higher $\theta$ value (45\%). Such significant change in $\theta$ is not acceptable, and we concluded that the scaling law is not applicable with these particular datasets. Other exceptions include water in regular MCM-41 material [23], where we simply could not extract physically acceptable $\theta$ and $D_{c}$ since the $D_{r}$ values reported there appear to be larger than $D_{\text {bulk }}$. At present, we do not fully understand the origins of these limitations. We are confident that it is pure coincidence that these two exceptions (and possibly others from other instruments) are for data measured on an instrument, for which we verified the law in several other cases. Possible answers may be found in the various method used to analyze the experimental data and/or could be associated with instrument characteristics. 


\section{Data treatment and Instrument Resolution}

In light of the observations above, it is evident that the diffusion coefficient values are indeed dependent on the energy resolution, the accessible $Q$-range and the model considered. Therefore, it is much more informative to examine emerging trends or characteristic features, instead of actual values, when comparing results from different sources. Because Eq. 1 does not explicitly include resolution, it is not possible to evaluate its effects based on the observed fitted parameters. However since energy resolution is key for experimentally determining $D_{r}$ on a given instrument, we naturally expect it to have an impact on the actual magnitude of $D_{c}$ associated with a given hydrated substrate. Also, having verified Eq. 1 with results obtained from a couple of standard models used to analyze QENS data (Lorentzian (LOR.) or the Kohlrausch-Williams-Watts (KWW) stretched exponential), we can not draw any direct connection between the fitting models and the applicability of Eq. 1 to given $D_{r}$ values. To put these models in perspective, we explicitly write their analytical expressions below. The LOR. case generally depicts a situation where the observed water dynamics is dominated by a single relaxation process. This intrinsic LOR. scattering function $S_{L O R}(Q, E)$ is generally defined as,

$$
S_{L O R}(Q, E)=\frac{1}{\pi} \frac{\Gamma(Q)}{\Gamma^{2}(Q)+E^{2}}
$$

When on the other hand, a distribution of relaxation processes is required to capture the experimental data, the KWW is a more appropriate model to use. This model function is defined as,

$$
S_{K W W}(Q, E)=\frac{1}{2 \pi} \int_{-\infty}^{\infty} d t \exp (-i E t / \hbar) \exp (t / \tau(Q))^{\beta(Q)}
$$

In addition to appropriately accounting for background signal, a proper convolution of the elastic signal plus either the LOR. or the KWW model with the instrument resolution function is necessary to fully describe QENS data. For further details on the subject, we refer the reader to [37].

Going back to the work of Takahara et al. [21], in which various models were used to analyze water dynamics in MCM-41, we find generally that $\theta$ always decreases with increasing pore size independently of the model, but that the actual magnitude is model dependent. For example, their KWW fits leads to a $\theta$ of $45 \%$ compared to $52 \%$ in the LOR. case for the $21.4 \AA$ 
pore diameter. These values decrease to $\theta=44 \%$ and $34 \%$ respectively in the $28.4 \AA$ pore diameter.

In order to overcome potential biases associated with instrumentation and/or data analysis, a reliable comparative study has to ideally be performed using data exclusively collected on the same exact neutron instrument, and treated using the same analytical approach. This is of course an ambitious and challenging endeavor, which is not practically feasible on the short term, as it would require several years to complete. This limitation arises because the access to neutron spectrometers worldwide is limited and restricted to large scale facilities where only a few experiments can be accommodated per calendar year. This is why we decided to base this study on available data in the literature collected over decades, with the goal of identifying common or unique characteristics in the dynamics of water in confinement.

\section{Exact analytical models}

To gain additional insights and better understanding of the variation of $\theta$ with the confinement geometry and the hydration amount as discussed respectively in sections 4 and 2, we turn to using exact analytical methods to describe water adsorption in three ideal standard geometries, respectively slit-type (intercalated planes), cylinder, and sphere, each with confinement diameter $d$. In these exact models, we assume (1) that only the first water layer is tightly bound to the wall of the confining surface (monolayer coverage with thickness $d_{c}=\sim 3.4 \AA$ and volume $V_{c}$ ), and (2) that the density of the water molecules is constant across the whole confining volume $V$ for simplicity. With these assumptions, the parameter $\theta$ can be expressed in terms of the variable $\Phi=\left(R_{\text {fill }} / R\right)$, where $R_{\text {fill }}$ is the filled water thickness (defined from the surface wall), and $R$ the characteristic confinement length $R=d / 2$, where $d$ is illustrated in Fig. 3 for the three geometries considered. The critical filling fraction for monolayer completion, below which $\theta=1$, can be expressed in terms of characteristic constant $\Phi_{c}=d_{c} / R$, for a fixed $d_{c}$ and $R$ values. The explicit expressions for $\theta$ versus $\Phi$ for the various geometries considered are listed in table 3 .

The left panel of Fig. 3 depicts the variation of $\theta$ with $\Phi$ for varying cylindrical pore sizes (with diameter $(d=2 R) 10,25,50$ and $100 \AA$ ) and the right panel shows the effect of changing geometries given a confinement dimension $R=12.5 \AA$. These calculations confirm the observed behavior 

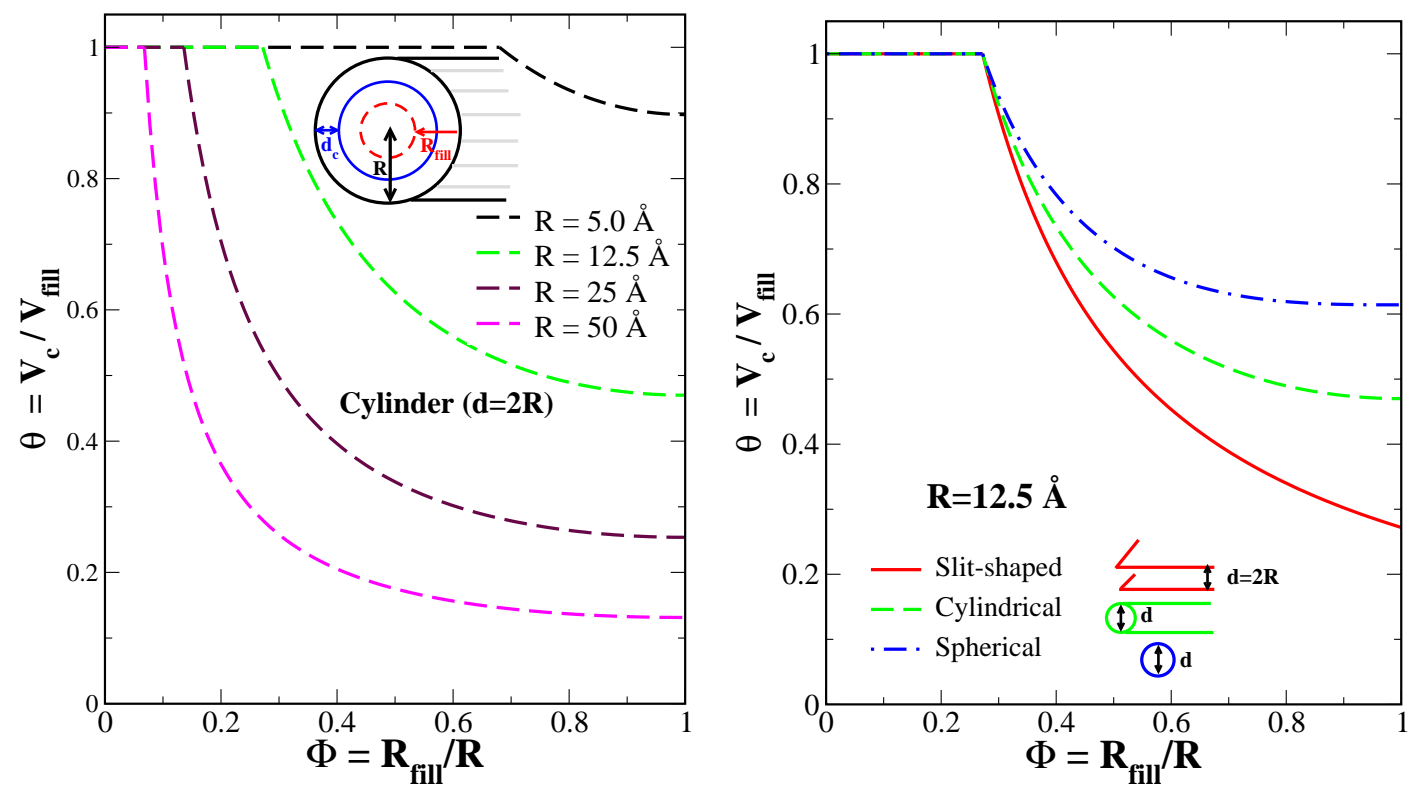

Figure 3: Left panel: Relative fraction of water molecules that are closest to the confining surface $\theta=V_{c} / V_{\text {fill }}$, where $V_{c}$ characterizes the volume occupied by the first adsorbed monolayer with characteristic thickness $d_{c}=3.4 \AA$ ) versus the filling parameter $\Phi=$ $\left(R_{f i l l} / R\right)$ in a cylindrical pore with diameter $d=2 R$. Right panel: Variation of $\theta$ with $\Phi$ for the various geometries with $R$ fixed at $12.5 \AA$. The characteristic dimension $d=2 R$ is defined here as the confining diameter in the cylindrical (1D) and spherical (3D) cases, but as the spacing gap for the slit geometry (2D), as illustrated. There is no corresponding visual interpretation for $d$ in the case of a flat surface.

of $\theta$ with increased pore size and hydration level. They also clearly clarify the influence of geometry, revealing the observed decrease of $\theta$ from 3D to lower dimension confinement at full filling. They also show that a lower $\theta$ is to be expected in the 2D case over any other dimensionality of comparable nanoconfinement gap above monolayer coverage. At full filling (or $\Phi=1$ ) in a restricted environment with $R=12.5 \AA$, we get $\theta$ values of $61 \%, 47 \%$ and $27 \%$ respectively for the sphere, cylinder and slit geometry ( see Fig. 3). By comparing these exactly calculated $\theta$ values with those inferred from Eq.1 using the neutron data, it is possible to verify the true nature of the surface geometry and predict the diffusive behavior of water.

Although these simple geometrical models provide an excellent description of the observed trends in the water adsorption behavior, they lack the accuracy that more realistic models used in MD simulations can achieve. 
Table 3: Parametric expressions of the fractional volume $\theta$ occupied by the first adsorbed water monolayer $\theta=\left(V_{c} / V_{\text {fill }}\right)$ versus the ratio $\Phi=\left(R_{\text {fill }} / R\right)$, where $R_{\text {fill }}$ and $R$ are respectively the characteristic filling thickness $\left(d_{\text {fill }}=2 R_{\text {fill }}\right)$, and the total available confinement dimension $(d=2 R)$. The dimension $d=2 R$ is schematically illustrated in Fig. 3 for the different geometries. The critical filling ratio needed to saturate the $1^{\text {st }}$ monolayer is $\Phi_{c}=d_{c} / R$.

\begin{tabular}{|c|c|c|}
\hline Geometry & $0<\Phi_{\text {fill }} \leq \Phi_{c}$ & $\Phi_{c} \leq \Phi_{\text {fill }} \leq 1$ \\
\hline Slit & 1 & $\Phi_{c} \Phi^{-1}$ \\
\hline Cylinder & 1 & $\left(2 \Phi_{c}-\Phi_{c}^{2}\right)\left(2 \Phi-\Phi^{2}\right)^{-1}$ \\
\hline Sphere & 1 & $\left(3 \Phi_{c}-3 \Phi_{c}^{2}+\Phi_{c}^{3}\right)\left(3 \Phi-3 \Phi^{2}+\Phi^{3}\right)^{-1}$ \\
\hline
\end{tabular}

They do satisfactorily describe the experimental observations. We anticipate to get even better agreement if interactions between molecules and those between the molecules and the surface, the surface roughness, and other key materials properties, can be accounted for.

\section{Case Study: water inside folded mesoporous silica-based mate- rials FSM.}

\subsection{Sample and experiment details}

To further test Eq. 1, we applied it to our own QENS data of water confined in the nanopores of folded mesoporous silica materials (FSM), collected on the backscattering spectrometer BASIS at the Spallation Neutron Source [38]. The FSM silica materials resemble the well-known MCM-41 systems, and possess 1D-like cylindrical channels (with smoother interior surfaces than in MCM-41[39]) that are hexagonally packed. Their synthesis methods have been previously described elsewhere[39]. We use two different pore sizes (FSM-12 (16 $\AA$ ) and FSM-22 (39 $\AA)$ respectively) to examine how each of the characteristic parameters $\theta$ and $D_{c}$ gets affected by varying confinement dimension. It is worthwhile noting that unlike in FSM-12 the dynamics of water in the larger FSM-22 pores have not been previously studied, probably because of the much larger pore dimension. We provide some relevant sample characteristics for the reader in Table 4 . The fully hydrated samples ( $h=100 \%$, corresponding here to full pore fillings) were sealed using flat $\mathrm{Al}$ containers, and measured at the following three temperatures, $270 \mathrm{~K}, 260 \mathrm{~K}$ and $245 \mathrm{~K}$. Additional data were also taken with each sample cooled down to very low temperatures, $\sim 50 \mathrm{~K}$, in order to parametrize the instrument 


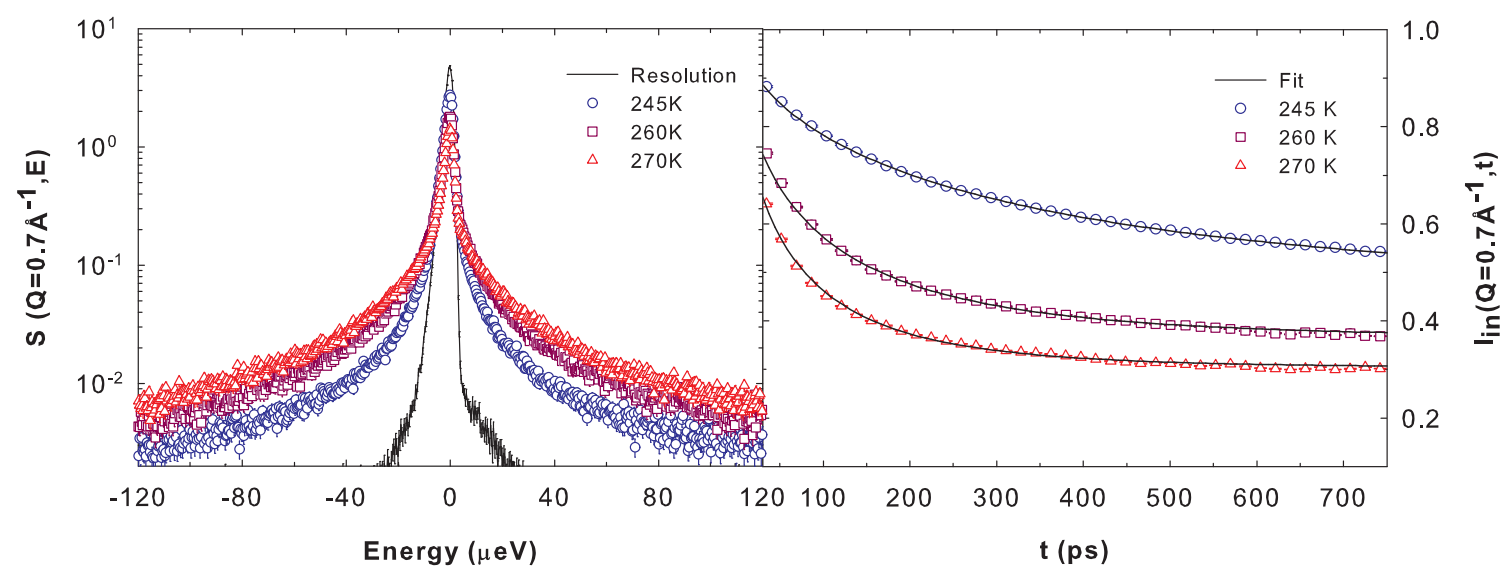

Figure 4: Left panel: Temperature dependence of the observed quasi-elastic neutron signal for FSM-12 at $Q=0.7 \AA^{-1}$. Right panel: Corresponding calculated intermediate scattering function $I_{i n}(Q, t)$.

Table 4: Characteristic diameter (Barrett-Joyner-Halenda (BJH) method [40]) and pore volume in FSM-12 and FSM-22, surface area (Brunauer-Emmett-Teller (SA BET))[39, 41]) and hydration level $h$ (weight $\%$ )

\begin{tabular}{|c|c|c|c|c|}
\hline Sample & diameter $(\AA)(\mathrm{BJH})$ & $\frac{V_{\text {pore }}}{V}(\%)$ & BET SA $\left(\mathrm{m}^{2} / \mathrm{g}\right)$ & $h$ (weight $\%)$ \\
\hline FSM-12 & 16 & 51 & 1130 & 100 \\
\hline FSM-22 & 39 & 96 & 1170 & 100 \\
\hline
\end{tabular}

resolution function, necessary for the data analysis. Elastic scattering data collected prior to the QENS measurements indicated that the adsorbed water in both FSM samples (despite the difference in their pore sizes) can be supercooled and does not freeze until very low temperatures (certainly above $230 \mathrm{~K})$. FSM-12 has naturally a much lower freezing temperature because of its smaller pore dimensions.

\subsection{Data analysis and results}

To analyze the data, we took the Fourier transform (FT) of the observed QENS spectra, as illustrated for example by Fig. 4 for FSM-12, to obtain the time dependent intermediate scattering function $I(Q, t)$ at each temperature. Such transformation is convenient, as it makes it possible to directly compare 
the measured intrinsic water dynamics with those from molecular dynamics simulations. This FT is carried out using,

$$
I(Q, t)=\frac{1}{2 \pi} \int_{-E_{m}}^{E_{m}} d E \exp (i E t / \hbar) S(Q, E)
$$

where $E_{m}$ is $\sim 120 \mu \mathrm{eV}$. The instrument contributions was then removed by dividing the resulting $I(Q, t)$ at each temperature by that corresponding to the $50 \mathrm{~K}$ data. These resolution free intrinsic $I_{i n}(Q, t)$ were then fitted in the time $t$ domain with a stretched exponential function for $t$ in the range $80 \leq t \leq 550 \mathrm{ps}$ for each of the $4 Q$ values, $0.3 \leq Q \leq 0.9 \AA^{-1}$, as highlighted by the solid lines in Fig. 4 for $Q=0.7 \AA^{-1}$. This time dependent model corresponds to,

$$
I_{\text {in }}(Q, t)=A_{0}+\left(1-A_{0}\right) \exp \left[-\left(\frac{t}{\tau}\right)^{\beta}\right]
$$

where $A_{0}$ represents the fraction of immobile water molecules (which corresponds to the EISF introduced above), $\tau$ is the relaxation time, and $\beta$ the stretching exponent. Given the limited statistical quality of our data and the faster rotational modes which complicate the fits at the high $Q$ (around and beyond $1 \AA^{-1}$ ), we kept $\beta$ fixed at its statistically averaged value $\langle\beta\rangle=0.64$ (determined from the first $3 Q$ using the $270 \mathrm{~K}$ data, for which the fits were excellent). This then allowed us to determine the dependency of each of the two fitting variables $A_{0}$, and $\tau$ on $Q$. By further inspecting these $Q$ dependences, valuable molecular dynamics details were extracted from the data. For example, we observed a mean relaxation time $\left\langle\tau_{\beta}\right\rangle=\frac{\tau}{\beta} \Gamma(1 / \beta)$ that exhibited a $Q$-dependence consistent with a translational jump diffusion, modeled with,

$$
\frac{1}{\left\langle\tau_{\beta}\right\rangle}=\frac{D_{r} Q^{2}}{1+D_{r} Q^{2}\left\langle\tau_{0}\right\rangle}
$$

where $D_{r}$ is the average translational diffusion coefficient of water inside the pores and $\left\langle\tau_{0}\right\rangle$ is the average time between jumps. $\Gamma(x)$ is the gamma function.

In Fig. 5, we display the observed $D_{r}(T)$ on a logarithmic scale as a function of $1000 / T$ to better highlight the difference in the water activation energy $E_{A}$ between the two confining media. Bulk water data is also shown for comparison [16]. The solid lines are Arrhenius fits to the observed 


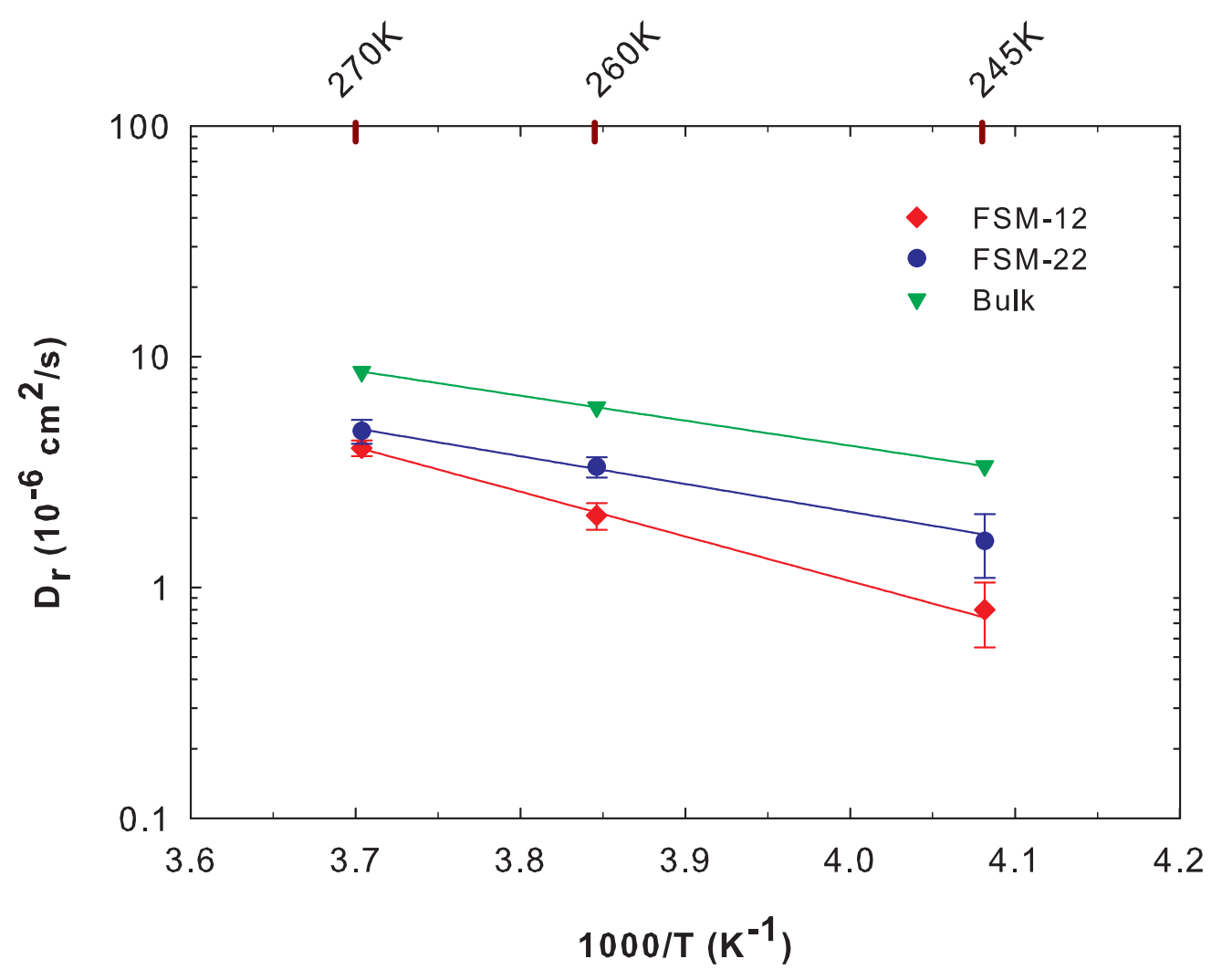

Figure 5: Observed diffusion coefficient $D_{r}(T)$ in FSM-12 (red solid diamonds) and in FSM-22 (blue solid circles) plotted as a function of 1000/T. Bulk water data are from Ref.[16]. 
values, leading respectively to $E_{A}=37 \mathrm{~kJ} / \mathrm{mol}$ in FSM-12 and $E_{A}=23$ $\mathrm{kJ} / \mathrm{mol}$ in FSM-22. These are to be compared with $E_{A} \simeq 21 \mathrm{~kJ} / \mathrm{mol}$ derived from the bulk water data [16] shown in the figure. In all subsequent analysis however, the bulk water $D_{b u l k}(T)$ is considered to be non-Arrhenius, as originally observed by Teixeira et al.. The fact that $E_{A}$ increases with decreasing pore diameter is to be expected since tighter confinement leads to an elevated barrier height for the H-bonds breaking. Due to the limited and somewhat lower $Q$-range used in the present analysis and the significant uncertainties associated with the resulting $\left\langle\tau_{0}\right\rangle$, it is much more difficult to interpret the temperature dependence of $\left\langle\tau_{0}\right\rangle$. The important feature in $\left\langle\tau_{0}\right\rangle$ is that it is consistently shorter in FSM-22 than in FSM-12 at all temperatures investigated, even with these uncertainties. Unlike $D_{r}(T)$, there is significant overlap between the two observed $\left\langle\tau_{0}\right\rangle$ even when the errorbars are considered. As a result of this, the determination of $E_{A}$ based on $\left\langle\tau_{0}\right\rangle(T)$ is inconclusive. This observation is similar to that of water in activated carbon materials [22]. Therefore, whenever we refer to $E_{A}$, we mean the $E_{A}$ determined from the temperature dependence of $D_{r}$.

Having determined $D_{r}$ of water in FSM-12 and FSM-22, we use them to test Eq. 1, as summarized in Fig. 6. The corresponding temperature independent parameters $\theta$ and $D_{c}$ are listed in Table 5. As documented earlier, we find again that $\theta$ decreases with increasing confinement size, yielding $\theta=61 \%$ for FSM-12 and $\theta=32 \%$ for FSM-22. Here, we notice a $D_{c}$ that is essentially zero and independent of the pore size. As indicated in Table 5 , the observed $\theta$ values agree reasonably well with those derived from the simplistic cylindrical model $\left(\theta_{\text {model }}\right)$ described in section 7 with $R=8$ and $19.5 \AA$ respectively, and full pore filling $\left(R_{\text {fill }}=R\right)$. From the expressions in table 3 , it is easy to see that $\theta$ converges to $2 \Phi_{c}-\Phi_{c}^{2}$ when $\Phi=1$, for a given cylindrical shape of pore radius $R$.

Furthermore, a comparison with the results obtained from the predominantly hydrophobic activated carbons (ACF) [22] reveals clear differences in the water-surface interactions between the two type of surfaces, marked by the much smaller $\theta$ values in ACF even at partial fillings.

Having found that $D_{c}=0$ is required for Eq. 1 to fit our FSM data collected on BASIS, we argue that more generally Eq.1 may be reduced to just $D_{r}(T) \simeq(1-\theta) D_{\text {bulk }}$ whenever $D_{c}$ becomes resolution limited on a neutron spectrometer. This implies that for most confining media a single parameter fit may be used to characterize data collected on instruments with even coarser resolution than BASIS in a systematic way such as examining 


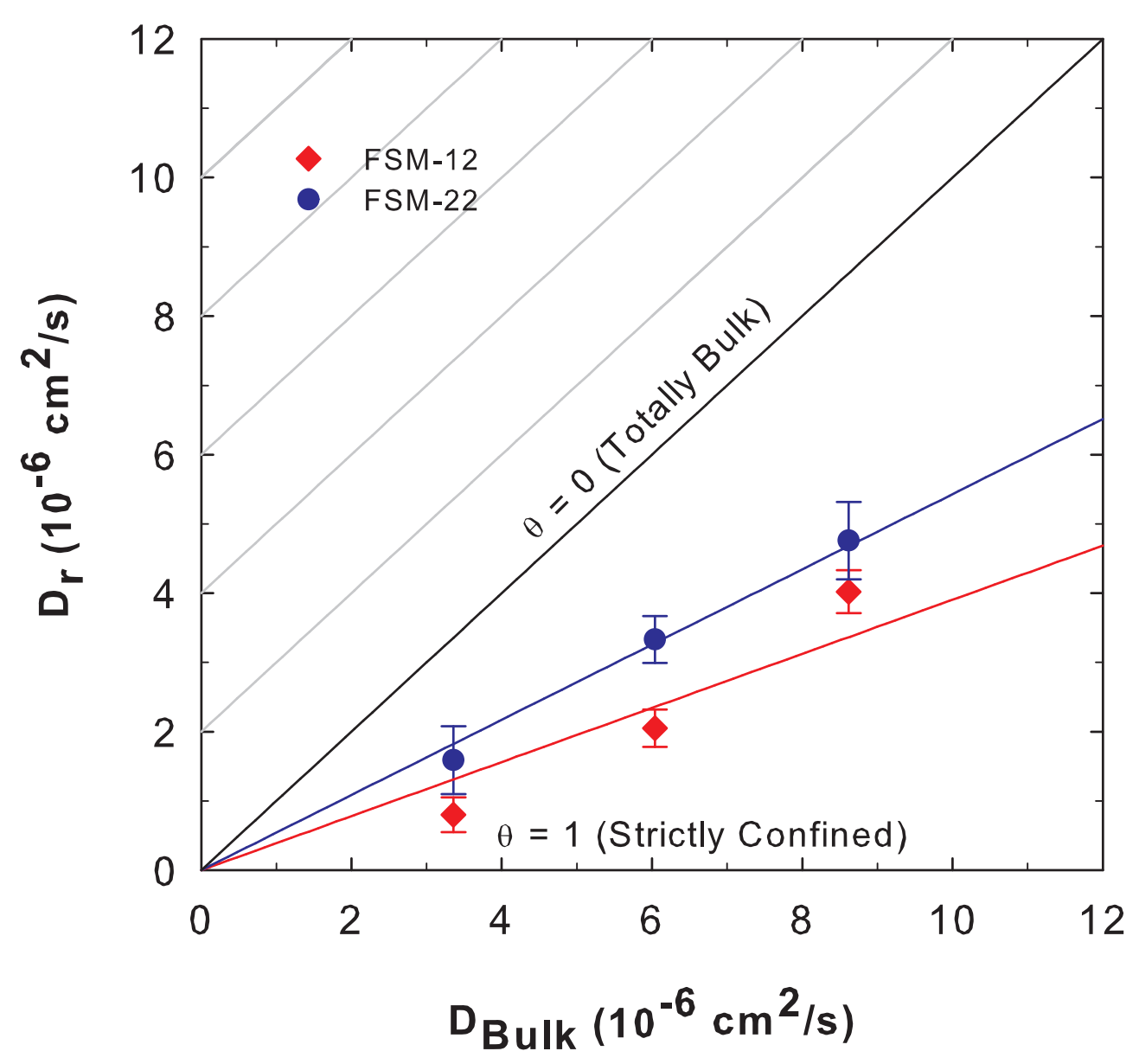

Figure 6: (Color online) Translational diffusion coefficient $\left(D_{r}\right)$ of water restricted within FSM-12 and FSM-22 at the three temperatures investigated versus bulk water coefficient $\left(D_{b u l k}\right)$. The solid lines represent the scaling behavior given by Eq. 1 , where $D_{c}$ is a temperature independent diffusion coefficient associated with the strictly confined water (i.e. most influenced by the pore walls), and $\theta$ the ratio between this water and the total water inside the pores. The top half of the graph with the solid grey lines indicates the limit where $D_{c}>D_{\text {bulk }}$, therefore not physically accessible within the present framework. Bulk water data are from Ref.[16] . 
Table 5: Observed temperature independent characteristic parameters of water confined in nanoporous silica FSM (fully filled); the fraction $\theta$ of water molecules closest to the pore walls $\left(\frac{N_{\text {bound }}}{N_{\text {total }}}\right)$, and their corresponding diffusion coefficients $D_{c}$. The analytically calculated $\theta_{\text {model }}$ values based on the cylindrical pore model described in section 7 are shown for comparison .

\begin{tabular}{|c|c|c|}
\hline Parameters & $\theta=\frac{N_{\text {bound }}}{N_{\text {total }}}$ & $\theta_{\text {model }}$ \\
\hline FSM-12 & $61 \%$ & $67 \%$ \\
\hline FSM-22 & $46 \%$ & $32 \%$ \\
\hline
\end{tabular}

water diffusion as a function of hydration level.

\section{Final remarks and conclusion}

In this study, we present a survey of a proposed predictive model for water transport, focusing on common features in different restricted environments. Based on our analysis, the following conclusions emerge:

- Water diffusion in confinement can be conveniently described using a sum of the contributions from molecules that act like bulk water, and those molecules (fraction $\theta$ ) that are strongly influenced by the confinement (interfacial water), which are experiencing a much slower diffusion at a rate $D_{c}$.

- For the scaling law to be physically meaningful, the following constraints $0 \leq \theta \leq 100 \%$ and $0 \leq D_{c} \leq D_{\text {bulk }}$, have to be met. The few observed violations of these rules are classified as exceptions, and attributed to possible variabilities in instrument characteristics and data analysis methods.

- For a given confinement size, $\theta$ increases and $D_{c}$ decreases with decreasing hydration level $h$ (or water coverage).

- Given a hydration amount, $\theta$ decreases and the net $D_{c}$ increases as the confinement size is enlarged.

- Excluding the influence of hydrophilicity, water mobility is also greatly impacted by the dimensionality it experiences under confinement. A 
generic feature is that $\theta$ is larger in $3 \mathrm{D}$ confinement (than in lower dimensions) because of the larger surface area. It does however decrease towards the bulk limit of 0 as the surface area is reduced and the hydration is increased towards full filling (i.e. becoming $100 \%$ bulk water).

Furthermore, we examined the effect of increasing pore size on the diffusive dynamics of water confined in folded-sheet mesoporous silica (FSM-12 (16 $\AA)$ and FSM-22 (39 $\AA)$ ) as a function of temperature. The quasi-elastic neutron scattering measurements show unambiguously that the smaller the pore size or the lower the temperature, the slower the dynamics become. The corresponding activation energy, based on the temperature dependence of the diffusion coefficient, is observed to increase with decreasing pore size, indicating that the energy barrier for diffusivity increases with tighter confinement. Using the scaling law, we determined the characteristics parameters $D_{c}$ and $\theta$ in the two FSM samples, and found that the $D_{c}$ values in these hydrophilic systems with tight pores to be effectively zero, unlike in systems with hydrophobic pore walls with comparable pore dimensions. We conclude thus in hydrophilic confinement with small pore size such as with the present FSM systems, the scaling law can be conveniently described using the sole geometrical parameter $\theta$, reducing it to $D_{r}(T) \simeq(1-\theta) D_{b u l k}$.

\section{Acknowledgments}

We are thankful to the staff at the Spallation Neutron Source (SNS), Oak Ridge National Laboratory (ORNL) for the support during the BASIS measurements. We acknowledge the use of the Mantid software package [42] for the data reduction and the DAVE software [43] for the data analysis. A.C. is grateful to the National GEM Consortium for its fellowship program. This research was supported as part of the Fluid Interface Reactions, Structures and Transport (FIRST) center, an Energy Frontier Research Center funded by the U.S. Department of Energy, Office of Basic Energy Sciences. Work at ORNL's SNS is sponsored by the Scientific User Facilities Division, Office of Basic Energy Sciences, U.S. Department of Energy.

[1] E. Chiavazzo, M. Fasano, P. Decuzzi, P. Asinari, Scaling behaviour for the water transport in nanoconfined geometries, Nature Communications 5 (4565) (2014) 1-11. doi:10.1038/ncomms4565. 
[2] J. Swenson, S. Cerveny, Dynamics of deeply supercooled interfacial water, Journal of Physics: Condensed Matter 27 (3) (2015) 033102. doi:10.1088/0953-8984/27/3/033102.

[3] R. Bergman, J. Swenson, Dynamics of supercooled water in confined geometry, Nature 403 (6767) (2000) 283-6. doi:10.1038/35002027.

[4] P. Gallo, F. Sciortino, P. Tartaglia, S.-h. Chen, Slow dynamics of water molecules in supercooled states, Physical Review Letters 76 (July 2015) (1996) 2730. doi:10.1103/PhysRevLett.76.2730.

[5] V. Crupi, D. Majolino, P. Migliardo, V. Venuti, U. Wanderlingh, T. Mizota, M. Telling, Neutron Scattering Study and Dynamic Properties of Hydrogen-Bonded Liquids in Mesoscopic Confinement. 2. The Zeolitic Water Case, The Journal of Physical Chemistry B 108 (14) (2004) 43144323. doi:10.1021/jp0371711.

[6] M. C. Bellissent-Funel, Status of experiments probing the dynamics of water in confinement, European Physical Journal E 12 (1) (2003) 83-92. doi:10.1140/epje/i2003-10023-9.

[7] I. Beta, J.-C. Li, M.-C. Bellissent-Funel, A quasi-elastic neutron scattering study of the dynamics of supercritical water, Chemical Physics 292 (2-3) (2003) 229-234. doi:10.1016/S0301-0104(03)00228-3.

[8] A. I. Kolesnikov, J.-m. Zanotti, C.-k. Loong, P. Thiyagarajan, C. J. Burnham, Anomalously soft dynamics of water in a nanotube : a revelation of nanoscale confinement, Physical Review Letters 93 (July) (2004) 035503. doi:10.1103/PhysRevLett.93.035503.

[9] A. Buchsteiner, A. Lerf, Water dynamics in graphite oxide Investigated with neutron scattering, Journal Physical Chemistry B 110 (2006) $22328-22338$.

[10] H. N. Bordallo, L. P. Aldridge, A. Desmedt, Water Dynamics in Hardened Ordinary Portland Cement Paste or Concrete : From Quasielastic Neutron Scattering, Journal Physical Chemistry B 110 (2006) 1796617976. 
[11] J.-C. Perrin, S. Lyonnard, F. Volino, Quasielastic neutron scattering study of water dynamics in hydrated nafion membranes, Journal of Physical Chemistry C 111 (8) (2007) 3393-3404. doi:10.1021/jp065039q.

[12] I. M. Briman, D. Rébiscoul, O. Diat, J.-M. Zanotti, P. Jollivet, P. Barboux, S. Gin, Impact of Pore Size and Pore Surface Composition on the Dynamics of Confined Water in Highly Ordered Porous Silica, Journal of Physical Chemistry C 116 (12) (2012) 7021-7028. doi:10.1021/jp3001898.

[13] R. Angelico, B. Balinov, a. Ceglie, U. Olsson, G. Palazzo, O. Soderman, Water Diffusion in Polymer-like Reverse Micelles. 2. Composition Dependence, Langmuir 15 (5) (1999) 1679-1684. doi:10.1021/la9813782.

[14] H. Yoo, R. Paranji, G. H. Pollack, Impact of Hydrophillic Surfaces on Interfacial Water Dynamics Probed with NMR Spectroscopy, Journal Physical Chemistry Letters 2 (6) (2011) 532-536. doi:10.1016/j.biotechadv.2011.08.021.Secreted.

[15] M. Sattig, M. Vogel, Dynamic Crossovers and Stepwise Solidi fi cation of Confined Water: A 2 H NMR Study, Physical Chemistry Letters 5 (2014) 174-178.

[16] J. Teixeira, M.-C. Bellissent-Funel, S.-H. Chen, A. Dianoux, Experimental determination of the nature of diffusive motions of water molecules at low temperatures, Physical Review A 31 (3) (1985) 1913.

[17] M.-C. Bellissent-Funel, S. H. Chen, J.-M. Zanotti, Single-particle space dynamics of water molecules in confined space, Physical Review E 51 (1995) 4558-4569.

[18] W. A. Kamitakahara, N. Wada, Neutron spectroscopy of water dynamics in NaX and NaA zeolites, Physical Review E - Statistical, Nonlinear, and Soft Matter Physics 77 (4) (2008) 1-10. doi:10.1103/PhysRevE.77.041503.

[19] S. Mitra, a. Pramanik, D. Chakrabarty, F. Jurányi, S. Gautam, R. Mukhopadhyay, Diffusion of water adsorbed in hydrotalcite: neutron scattering Study, Journal of Physics: Conference Series 92 (2007) 012167. doi:10.1088/1742-6596/92/1/012167. 
[20] Z. Wang, E. Fratini, M. Li, P. Le, E. Mamontov, P. Baglioni, S.-H. Chen, Hydration-dependent dynamic crossover phenomenon in protein hydration water, Physical Review E 90 (4) (2014) 1-5. doi:10.1103/PhysRevE.90.042705.

[21] S. Takahara, N. Sumiyama, S. Kittaka, T. Yamaguchi, M.-C. BellissentFunel, Neutron scattering study on dynamics of water molecules in MCM-41. 2. Determination of translational diffusion coefficient., The journal of physical chemistry. B 109 (22) (2005) 11231-11239. doi:10.1021/jp046036l.

[22] S. O. Diallo, Pore-size dependence and characteristics of water diffusion in slitlike micropores, Physical Review E - Statistical, Nonlinear, and Soft Matter Physics 012312 (April) (2015) 1-10. doi:10.1103/PhysRevE.92.012312.

[23] S.-H. Chen, F. Mallamace, C.-Y. Mou, M. Broccio, C. Corsaro, A. Faraone, L. Liu, The violation of the Stokes-Einstein relation in supercooled water., Proceedings of the National Academy of Sciences of the United States of America 103 (35) (2006) 12974-12978. doi:10.1073/pnas.0603253103.

[24] M.-C. Bellissent-Funel, J. Teixeira, K. F. Bradley, S. H. Chen, Dynamics of hydration water in protein, Journal de Physique I 2 (6) (1992) 9951001. doi:10.1051/jp1:1992192.

[25] M.-C. Bellissent-Funel, J.-M. Zanotti, S. H. Chen, Slow dynamics of water molecules on the surface of a globular protein, Faraday Discussions 103 (1996) 281. doi:10.1039/fd9960300281.

[26] S. Khodadadi, J. H. Roh, a. Kisliuk, E. Mamontov, M. Tyagi, S. a. Woodson, R. M. Briber, a. P. Sokolov, Dynamics of biological macromolecules: not a simple slaving by hydration water, Biophysical Journal 98 (7) (2010) 1321-1326. doi:10.1016/j.bpj.2009.12.4284.

[27] J. D. Nickels, H. O'Neill, L. Hong, M. Tyagi, G. Ehlers, K. L. Weiss, Q. Zhang, Z. Yi, E. Mamontov, J. C. Smith, A. P. Sokolov, Dynamics of protein and its hydration water: neutron scattering studies on fully deuterated GFP, Biophys J 103 (7) (2012) 1566-1575. doi:10.1016/j.bpj.2012.08.046. 
[28] F. González Sánchez, F. Jurányi, T. Gimmi, L. V. Loon, T. Seydel, T. Unruh, Dynamics of supercooled water in highly compacted clays studied by neutron scattering, Journal of Physics: Condensed Matter 20 (41) (2008) 415102. doi:10.1088/0953-8984/20/41/415102.

[29] J. Swenson, R. Bergman, W. S. Howells, Quasielastic neutron scattering of two-dimensional water in a vermiculite clay, Journal of Chemical Physics 113 (7) (2000) 2873-2879. doi:10.1063/1.1305870.

[30] E. Mamontov, C. J. Burnham, S. H. Chen, a. P. Moravsky, C. K. Loong, N. R. De Souza, a. I. Kolesnikov, Dynamics of water confined in single- and double-wall carbon nanotubes, Journal of Chemical Physics 124 (19). doi:10.1063/1.2194020.

[31] Y. Liu, Q. Wang, T. Wu, L. Zhang, Fluid structure and transport properties of water inside carbon nanotubes., The Journal of chemical physics 123 (23) (2005) 234701. doi:10.1063/1.2131070.

[32] A. Paciaroni, M. Casciola, E. Cornicchi, M. Marconi, G. Onori, M. Pica, R. Narducci, Temperature-dependent dynamics of water confined in nafion membranes., The journal of physical chemistry. B 110 (28) (2006) 13769-13776. doi:10.1021/jp0572176.

[33] T. L. Spehr, B. Frick, M. Zamponi, B. Stühn, Dynamics of water confined to reverse AOT micelles, Soft Matter 7 (12) (2011) 5745. doi:10.1039/c1sm05204g.

[34] H. Li, E. Fratini, W. S. Chiang, P. Baglioni, E. Mamontov, S. H. Chen, Dynamic behavior of hydration water in calcium-silicate-hydrate gel: A quasielastic neutron scattering spectroscopy investigation, Physical Review E - Statistical, Nonlinear, and Soft Matter Physics 86 (6) (2012) 1-7. doi:10.1103/PhysRevE.86.061505.

[35] T. Yamada, R. Yonamine, T. Yamada, H. Kitagawa, M. Tyagi, M. Nagao, O. Yamamuro, Quasi-elastic neutron scattering studies on dynamics of water confined in nanoporous copper rubeanate hydrates., The journal of physical chemistry. B 115 (46) (2011) 13563-9. doi:10.1021/jp2029467.

[36] A. Faraone, K.-H. Liu, C.-Y. Mou, Y. Zhang, S.-H. Chen, Single particle dynamics of water confined in a hydrophobically modified MCM-41-S 
nanoporous matrix., The Journal of chemical physics 130 (13) (2009) 134512. doi:10.1063/1.3097800.

[37] M. Bee, Quasielastic Neutron Scattering, Adam Hilger, Bristol, 1988.

[38] E. Mamontov, K. W. Herwig, A time-of-flight backscattering spectrometer at the Spallation Neutron Source, BASIS, Review of Scientific Instruments 82 (8). doi:10.1063/1.3626214.

[39] S. Inagaki, Y. Fukushima, K. Kuroda, Synthesis of highly ordered mesoporous materials from a layered polysilicate, Journal of the Chemical Society, Chemical Communications (8) (1993) 680. doi:10.1039/c39930000680.

[40] E. P. Barrett, L. G. Joyner, P. P. Halenda, TheDetermination of Pore Volume and Area Distributions in Porous Substances. I. Computations from Nitrogen Isotherms", Journal of American Chemical Society 73 (1948) (1951) 373-380.

[41] S. Brunauer, P. H. Emmett, E. Teller, Adsorption of gases in multimolecular layers, Journal of the American Chemical Society 60 (1) (1938) 309-319. doi:4074706.

[42] O. Arnold, J. C. Bilheux, J. M. Borreguero, a. Buts, S. I. Campbell, L. Chapon, M. Doucet, N. Draper, R. Ferraz Leal, M. a. Gigg, V. E. Lynch, a. Markvardsen, D. J. Mikkelson, R. L. Mikkelson, R. Miller, K. Palmen, P. Parker, G. Passos, T. G. Perring, P. F. Peterson, S. Ren, M. a. Reuter, a. T. Savici, J. W. Taylor, R. J. Taylor, R. Tolchenov, W. Zhou, J. Zikovsky, Mantid - Data analysis and visualization package for neutron scattering and $\mu$ SR experiments, Nuclear Instruments and Methods in Physics Research, Section A: Accelerators, Spectrometers, Detectors and Associated Equipment 764 (2014) 156166. doi:10.1016/j.nima.2014.07.029.

[43] R. T. Azuah, L. R. Kneller, Y. Qiu, P. L. W. Tregenna-Piggott, C. M. Brown, J. R. D. Copley, R. M. Dimeo, DAVE: A Comprehensive Software Suite for the Reduction, Visualization, and Analysis of Low Energy Neutron Spectroscopic Data, Journal of Research of the National Institute of Standards and Technology 114 (6) (2009) 341. doi:10.6028/jres.114.025. 